\title{
Flexible SERS active detection from novel Ag nano-necklaces as highly reproducible and ultrasensitive tips
}

\author{
Chunyang Duan ${ }^{1}$, Bei Ren ${ }^{1,2}$, Hongying Liu ${ }^{2}$, Yu Wang ${ }^{1 *}$ and Yunfa Chen ${ }^{1}$
}

\begin{abstract}
Surface-enhanced Raman scattering (SERS) spectroscopy has been considered as a promising way to realize real-time, in-situ and ultrasensitive analysis of chemoand biochemical molecules in different applications even in intracellular or aqueous environments. In this work, polymer-supported novel Ag nano-necklaces (AgNLs) as flexible SERS substrates were fabricated for ultrasensitive chemical and biological detection. With the stringing of dense "hot spot" in three-dimension, AgNLs located on polydimethylsiloxane (PDMS) work like the removable and reusable "tip" on the surface of analytes with different morphologies and conditions. The novel substrate shows ultra-high enhancement factor (as high as $10^{9}$ ) with excellent reproducibility and long-term stability ( 7 months) in an aqueous environment. With further functionalizing with $p$-mercaptobenzoic acid, AgNLs/PDMS elastomer also reveals sensitive and consistent $\mathrm{pH}$ detection ability over the wide range of $\mathrm{pH}$ 4.0-9.0, indicating their wide applications in biological and environmental fields. This work provides a feasible strategy for designing ultrasensitive, reproducible and flexible SERS substrate for practical detection.
\end{abstract}

Keywords: Ag nano-necklace, SERS, flexible substrate

\section{INTRODUCTION}

In recent years, surface-enhanced Raman scattering (SERS) spectroscopy garnered intense attention for its ability of real-time, in-situ and ultrasensitive analysis of chemo- and biochemical molecules in different applications even in intracellular or aqueous environments [1-3]. Thanks to the discovery of "hot spots" which congregate high density of electromagnetic field in the gaps between the two adjacent coupling metal nanoparticles with distance on the order of a few nanometers, currently, SERS substrates based on plasmonic metal nanostructures have been used to detect protein, DNA, toxic molecules and other chemical or biological species even on molecular-level [4-7]. However, further application of SERS detection has been challenging due to the problems like poor maneuverability, retrievability of the SERS substrates and the low reproducibility of the measurements.

In order to obtain high enhancement factor (EF), ordinary SERS substrates are mainly based on colloidal silver nanoparticles (AgNPs) or their assemblies on nanowires or two-dimensional nanosheets such as graphene. The randomly achieved "hot spots" through the aggregation of the nanoparticles frequently lead to unrepeatable Raman signals; meanwhile AgNPs often suffer from dramatic morphological changes under irradiation or aqueous environments [8-10]. Modifying AgNPs with protection layers can efficiently improve their stability $[11,12]$; however, the protection layer might severely weaken the substrates' sensitivity and introduce noise signals. The other strategy is to orderly arrange noble metal particles into closely packed periodic patterns through lithographic methods $[13,14]$. Unfortunately, current lithographic facilities still have difficulties in achieving sub-10-nm gaps between the particles. Meanwhile the tedious preparation and implementation steps of SERS substrates are too complicated for their practical applications. The other issue that hinders the application of SERS detection is the compatibility of the SERS substrates. Lithographic periodic metal patterns are often constructed on brittle and rigid substrates which are incompatible with multiple sample matrices [15]. Spreading

\footnotetext{
${ }^{1}$ State Key Laboratory of Multiphase Complex Systems, Institute of Process Engineering, Chinese Academy of Sciences, Beijing 100190, China

${ }^{2}$ School of Chemical and Environmental Engineering, China University of Mining and Technology (Beijing), Beijing 100083, China

${ }^{*}$ Corresponding author (email: wyu@ipe.ac.cn)
} 
plasmonic metal structures on the surface of analytes is another method to realize SERS detection on different sample bases such as fruits and fluids, however, in this way the SERS substrates can hardly be recycled and the surfaces of analytes are contaminated. Therefore, facile fabrication of high sensitive SERS substrates with both high compatibility and reproducibility still remains a challenge.

Inspired by the measuring method of tip-enhanced Raman spectroscopy (TERS) $[16,17]$, we proposed a new type of SERS substrate that could work like the removable and reusable "tip" on the surface of analytes with different morphologies and conditions. This original SERS substrate combines novel plasmonic metal nanostructures with highly sensitive SERS response and the transparent and flexible polymer film holder as an elastomer compatible to different surfaces. During the detection, the film could be pasted on different sample matrix with different morphology and the plasmonic metal-nanostructured "tip" would reach the analyte. After the measurement, the SERS substrates could be removed from the surface of matrix and rinsed for the further use just like "post-it note", indicating good recyclability.

Based on the above strategy, a highly sensitive SERS substrate with high reproducibility was designed by fixing the novel Ag nano-necklaces (AgNLs) on the surface of polydimethylsiloxane (PDMS) film. To be specific, Ag nanonecklace (AgNL) used as SERS-active "tip" with high-density "hot spots" spread in three-dimension was fabricated by sacrificing Si nanowires (SiNWs) templates. Although SERS substrates based on plasmonic metal nanoparticles decorated SiNWs have been studied and published, AgNLs fabricated by sacrificing SiNWs templates have entirely different morphology with high originality and unique properties and have not been reported previously. PDMS film is chosen as the supporting base due to its flexibility and transparency which can be easily pasted on the surface of the tested object without affecting the incident laser and corresponding Raman signals [18]. Due to the novel design and AgNLs' unique structure, the flexible SERS substrates exhibit ultrahigh sensitivity even up to the single-molecule level, good detection stability in aqueous environment and excellent spot-to-spot reproducibility. Furthermore, with the further functionalizing by $\mathrm{pH}$-sensitive $\mathrm{p}$-mercaptobenzoic acid (pMBA) molecules, the AgNLs/PDMS elastomer also demonstrates sensitive and consistent $\mathrm{pH}$ detection ability over the wide range of $\mathrm{pH} 4.0-9.0$. These results indicate that the AgNLs/PDMS elastomer has great potential for sensitive, recyclable and versatile detection of chemical or biological substances in practical applications.

\section{EXPERIMENTAL SECTION}

\section{Preparation of AgNLs/PDMS films}

SiNWs were synthesized by thermal evaporation of $\mathrm{SiO}$ powder with $\mathrm{Sn}$ as catalyst in a home-built furnace following the vapor-liquid-solid (VLS) process. Before using, SiNWs were firstly etched in $5 \% \mathrm{HF}(v / v)$ solution for 30 min to remove the $\mathrm{SiO}_{2}$ shell and obtain $\mathrm{H}$-terminated surfaces. As HF is a highly corrosive acid, it should be handled with caution. PDMS elastomer was prepared by curing the mixture of Sylgard 184 silicone rubber and curing agent $(w / w=10: 1)$ at $80^{\circ} \mathrm{C}$ for $12 \mathrm{~h}$. After the synthesis of SiNWs and PDMS film, SiNWs were transferred onto PDMS by face-to-face transfer process: firstly, SiNWs were dispersed in ethanol. With ultrasonic process for 10 min to improve the dispersion, the mixture was carefully dropped along the beaker wall into the surface of deionized (DI) water which occupies $2 / 3$ of the beaker. Due to the concentration and surface tension difference of water and ethanol, SiNWs would float on the surface of the mixed liquid. Then, a pre-cleaned glass slide was used to fish out the SiNWs film on the surface of the solution and dried with $\mathrm{N}_{2}$ flow. Finally, the PDMS film is placed on the glass slide which covered by SiNWs to transfer the SiNWs to the surface of PDMS due to the strong adhesion between them.

When synthesizing AgNLs/PDMS film, etchants that contains the mixture of $\mathrm{HF}\left(4.8 \mathrm{~mol} \mathrm{~L}^{-1}\right)$ and $\mathrm{AgNO}_{3}(5$ $\mathrm{mmol} \mathrm{L}{ }^{-1}$ ) were firstly heated to $50^{\circ} \mathrm{C}$, then, in the water bath with $50^{\circ} \mathrm{C}$ the preheated etchant $\left(\mathrm{HF} / \mathrm{AgNO}_{3}\right)$ was rapidly added on the surface of SiNWs/PDMS film. After allowing the reaction to proceed for $3 \mathrm{~min}$, the film was quickly taken out from the etchant and rinsed with DI water and ethanol twice each, sequentially. Meanwhile, AgNLs could be synthesized through the similar procedure, in which the oxide layer on SiNWs (about $1.0 \mathrm{mg}$ ) were also removed by HF (5\%), then H-SiNWs were dispersed in $1 \mathrm{~mL}$ of ethanol homogeneously after ultrasonic process for $5 \mathrm{~min}$. Then, in the water bath with $50^{\circ} \mathrm{C}$ the preheated etchant $\left(\mathrm{HF} / \mathrm{AgNO}_{3}\right)$ was rapidly added to $1 \mathrm{~mL}$ of SiNW-ethanol suspension. After allowing the reaction to proceed for $3 \mathrm{~min}$, the solution was quickly centrifuged at $8000 \mathrm{rpm}$ for $2 \mathrm{~min}$, and the precipitate was rinsed with DI water and ethanol twice each, sequentially. Finally, the AgNLs were dispersed in ethanol for further use.

\section{SERS detection of R6G with AgNLs/PDMS film}

All of the Raman spectra were collected using a Renishaw invia Raman confocal microscope with $50 \times$ objective, 532 $\mathrm{nm}$ wavelength excitation, $0.5 \mathrm{~mW}$ laser power, and $10 \mathrm{~s}$ 
acquisition time. During the tests, rhodamine 6G (R6G) with different concentration was dropped into the hemispherical dimple $(\Phi=16 \mathrm{~mm}, h=0.4 \mathrm{~mm})$ of the glass holder with full volume. Then AgNLs/PDMS film covered on the top of hemispherical dimple to contact the R6G solutions. Laser was shot to the AgNLs through PDMS film and the Raman signals were collected through the SERS substrate. The detecting spots were labeled by ink spots with a mark pen. After a test of the certain concentration, AgNLs/PDMS film was taken from the hemispherical dimple and its inner side contacting with R6G solution was washed with DI water and ethanol to remove the residue dye molecules, and then it was covered on the R6G solution with another concentration to investigate the SERS sensitivity. In order to make sure the entirely removal of residue R6G molecules in the SERS substrate, Raman spectra was measured to check whether R6G was still adsorbed on AgNLs after the washing. With the ink mark on the top side of AgNLs/PDMS film, the same AgNL could be found even the R6G solution was changed. For the reproducibility tests, five different AgNLs/PDMS films were used to detect R6G solution with the concentration of $1 \times 10^{-9} \mathrm{~mol} \mathrm{~L}^{-1}$, and six different spots were investigated for each AgNLs. In order to investigate the stability of the SERS substrate, after the study of reproducibility, the SERS active samples were firstly stored in DI water for 7 months. Then, 35 spots chosen from five different AgNLs/PDMS substrates were investigated under the same condition.

\section{PH detection with pMBA-AgNLs/PDMS film}

AgNLs/PDMS films were firstly exposed to an aqueous solution of pMBA $\left(1 \times 10^{-5} \mathrm{~mol} \mathrm{~L}^{-1}\right)$ for $1 \mathrm{~h}$ to ensure full surface coverage of AgNLs. Before the Raman measurements, the samples were thoroughly rinsed with DI water. The testing solutions with different $\mathrm{pH}$ values were prepared with phosphate-buffered saline (PBS) and a Mettler Toledo portable $\mathrm{pH}$ meter was used to adjust the $\mathrm{pH}$ of the buffer solution. The experiments were conducted under $532-\mathrm{nm}$ laser with $50 \times$ objective, $0.5 \mathrm{~mW}$ laser power, and $30 \mathrm{~s}$ acquisition time. During the measurements, the PBS solutions with different $\mathrm{pH}$ values were firstly dropped into the hemispheric dimple of our home-build sample holder with the full volume. Then pMBA-AgNLs/PDMS film contacted the testing solution with different $\mathrm{pH}$ by covering the solution-filled hemispheric dimple. Ink spots were used to label the detection point. After testing in solution with a certain $\mathrm{pH}$ value, $\mathrm{pMBA}-\mathrm{AgNLs} / \mathrm{PDMS}$ film was peeled from the hemispheric dimple and rinsed with DI water, after drying in $\mathrm{N}_{2}$ flow; it was stick to the surface of hemispheric dim- ple filled with liquids with another $\mathrm{pH}$ value. For the $\mathrm{pH}$ tests in the range of 4.0 to 9.0, three pMBA-AgNLs/PDMS films were used for tests, and 10 testing spots from different AgNLs of each sample were studied. Meanwhile, in the reversible studies in acid ( $\mathrm{pH}$ 5.0) and alkaline ( $\mathrm{pH} 9.0)$ solutions, 10 testing spots from two pMBA-AgNLs/PDMS films were chosen for investigation and five cycles were conducted for each spots.

\section{RESULTS AND DISCUSSION}

Inspired by the metal nanoparticles' etching of $\mathrm{Si}$ wafer $[19,20]$, we assembled Ag nanoparticles into a necklace with dense "hot spots" spread in 3-dimension by sacrificing SiNWs templates. Meanwhile, due to the facile transfer and high adhesion between the SiNWs and flexible PDMS films, AgNLs could be located on the surface of PDMA on the fixed position. Fig. 1a illustrates the schematic of the fabrication procedure of the AgNLs/PDMS elastomer. After synthesizing PDMS film by curing the Sylgard 184 silicone rubber and SiNWs by VLS process [21], H-terminated SiNWs were deposited on the surface of PDMS film by face-to-face transfer to obtain the single layer covering of SiNWs (SI, S1) [22]. Then, in the water bath with $50^{\circ} \mathrm{C}$ the preheated etchant $\left(\mathrm{HF} / \mathrm{AgNO}_{3}\right)$ was rapidly added on the surface of SiNWs/PDMS film. With the adding of etchant, $\mathrm{Ag}^{+}$was reduced immediately by the highly reductive $\mathrm{Si}-\mathrm{H}$ to form dispersive AgNPs on the surface of SiNWs, which was similar to the ordinary synthesis of AgNPs decorated SiNWs. However, differing from the ordinary metallization of SiNWs, HF and $50^{\circ} \mathrm{C}$ water bath provide further chemical reactions in our system, in which SiNWs can be oxidized (the reaction as shown in R1) and further dissolved by HF. Meanwhile, the excess $\mathrm{Ag}^{+}$ions are subsequently reduced back to AgNPs as a result of the electronegativity and the electron transfer (R2). Thus, the AgNPs grow bigger and gradually encroach on the SiNWs. In the end, the templating SiNWs are vanished leaving the finite AgNPs stick together like beads of the necklaces. The gradually vanishing of SiNWs could also be demonstrated through Raman and SEM of different reaction stage (SI, S2).

$$
\begin{gathered}
\mathrm{Si}+6 \mathrm{HF} \rightarrow \mathrm{H}_{2} \mathrm{SiF}_{6}+4 \mathrm{H}^{+}+4 \mathrm{e}^{-}\left[E^{0}=1.2 \mathrm{~V} / \mathrm{SHE}\right](\mathrm{R} 1) \\
\mathrm{Ag}^{+}+\mathrm{e}^{-} \rightarrow \mathrm{Ag}\left[E^{0}=0.79 \mathrm{~V} / \mathrm{SHE}\right]
\end{gathered}
$$

After allowing the reaction to proceed for $3 \mathrm{~min}$, the film was quickly taken out from the etchant and rinsed with DI water and ethanol, sequentially. As sacrificial templates, SiNWs determines the location and orientation of the final 

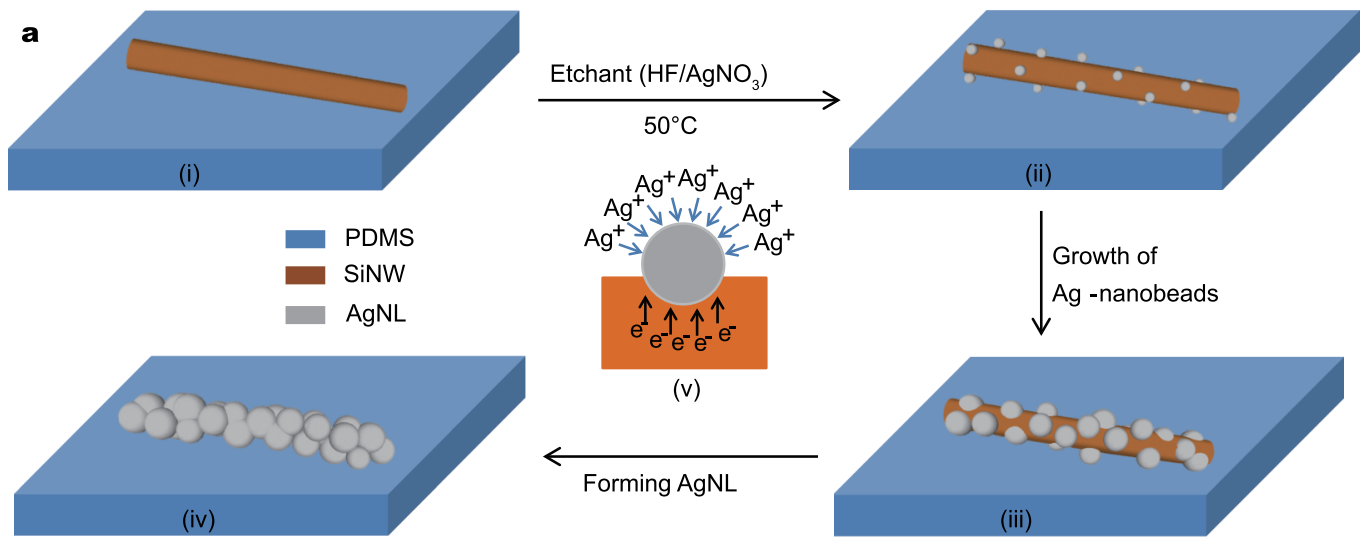

(v)

Forming AgNL
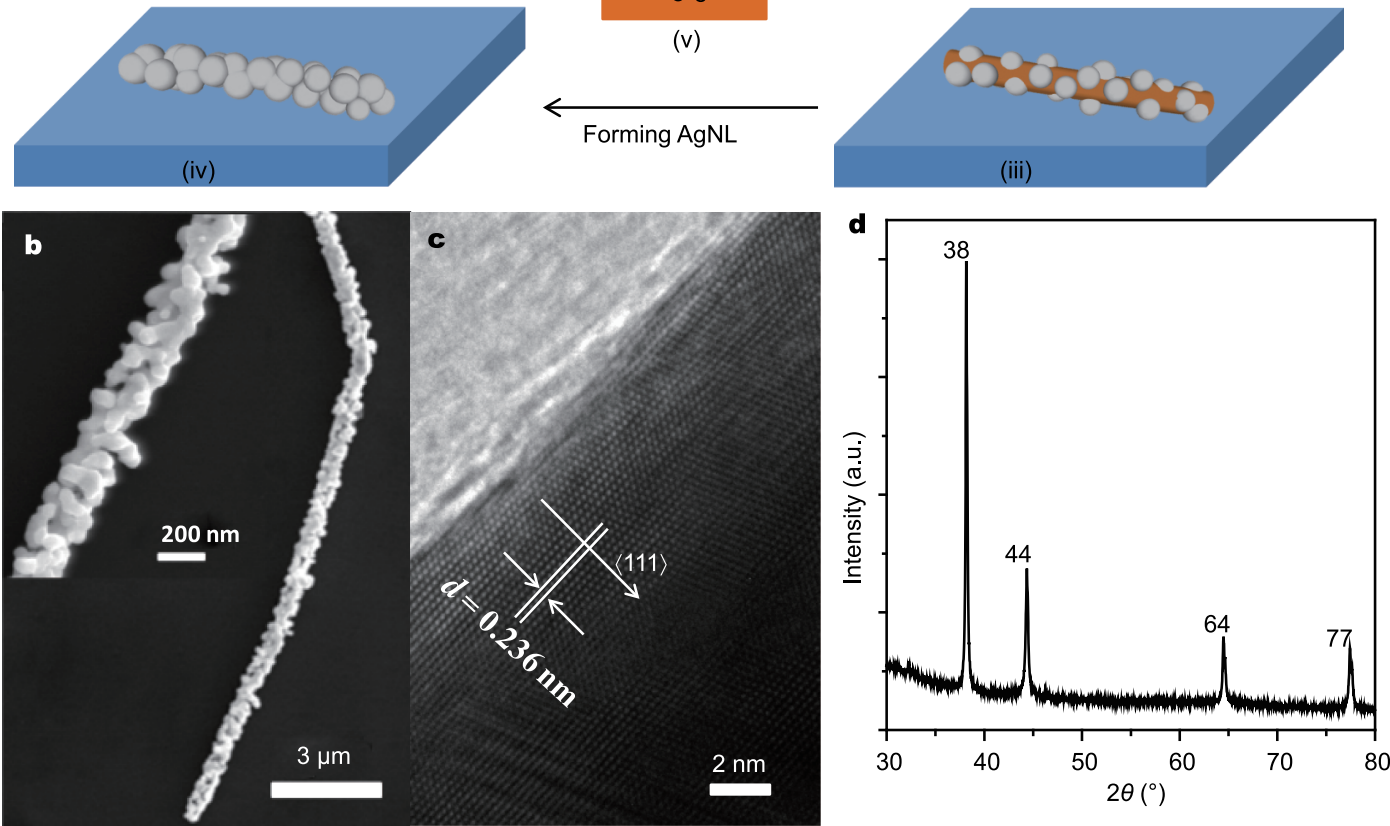

Figure 1 (a) Schematic of the fabrication procedure of the AgNLs/PDMS elastomer; SEM image (b), HRTEM image (c) and XRD results (d) of a typical AgNL.

AgNLs (SI, S3). Since the fabrication of SiNWs is well developed and the controlled transfer of SiNWs is obtained by contact printing, the site-specific synthesis of AgNLs or their arrays could also be realized [19-23].

Fig. $1 \mathrm{~b}$ shows the SEM image of an AgNL. It can be seen that Ag nano-beads stick together to form the nanonecklace with the gaps of sub $10 \mathrm{~nm}$ spreading in three-dimensional dispersion (inset of Fig. 1b) which creates the high-density packed "hot spots" along the AgNL. To obtain further insight into the AgNL, a high-resolution TEM (HRTEM) image taken from a typical area of the nanobeads was exhibited in Fig. 1c. The nano-beads of the AgNLs are composed of lattices with interplanar spacing of $0.236 \mathrm{~nm}$, corresponding to the $d$ spacing for the (111) planes of cubic structured Ag, which indicates the good crystallinity of these Ag nano-beads. Fig. 1d shows the corresponding XRD pattern of the as-prepared AgNLs. All the diffraction peaks are readily indexed to a cubic phase of $\mathrm{Ag}$ (JCPDS No. 65-2871) and no other characteristic peaks are observed for impurities, indicating the formation of pure cubic phase Ag with high crystallinity.

The high-resolution measurement of sensitivity and reproducibility of AgNLs/PDMS elastomer was performed with R6G as the model indicator. Schematic of the measurement setup is shown in Fig. 2a. In the experiment, R6 G with different concentration was dropped in the hemispherical dimple ( $\Phi=16 \mathrm{~mm}, h=0.4 \mathrm{~mm})$ of a custommade sample holder with full volume. Then, the hemispherical dimple was sealed by AgNL/PDMS film with the AgNLs contacted with R6G solutions (SI, Fig. S6). 532-nm laser was shot through the SERS substrate from the $50 \times$ objective lens and the Raman signals were collected by a Renishaw Raman spectrometer (Invia reflex Raman microscope). Similar to the light-scattering of the nanowires, AgNLs can be observed under microscope (SI, Fig. S5d), thus an ink spot can be made by mark pen to label the AgNLs under study (SI, S4). After a test of the certain concentration, AgNL/PDMS film was taken from the hemis- 

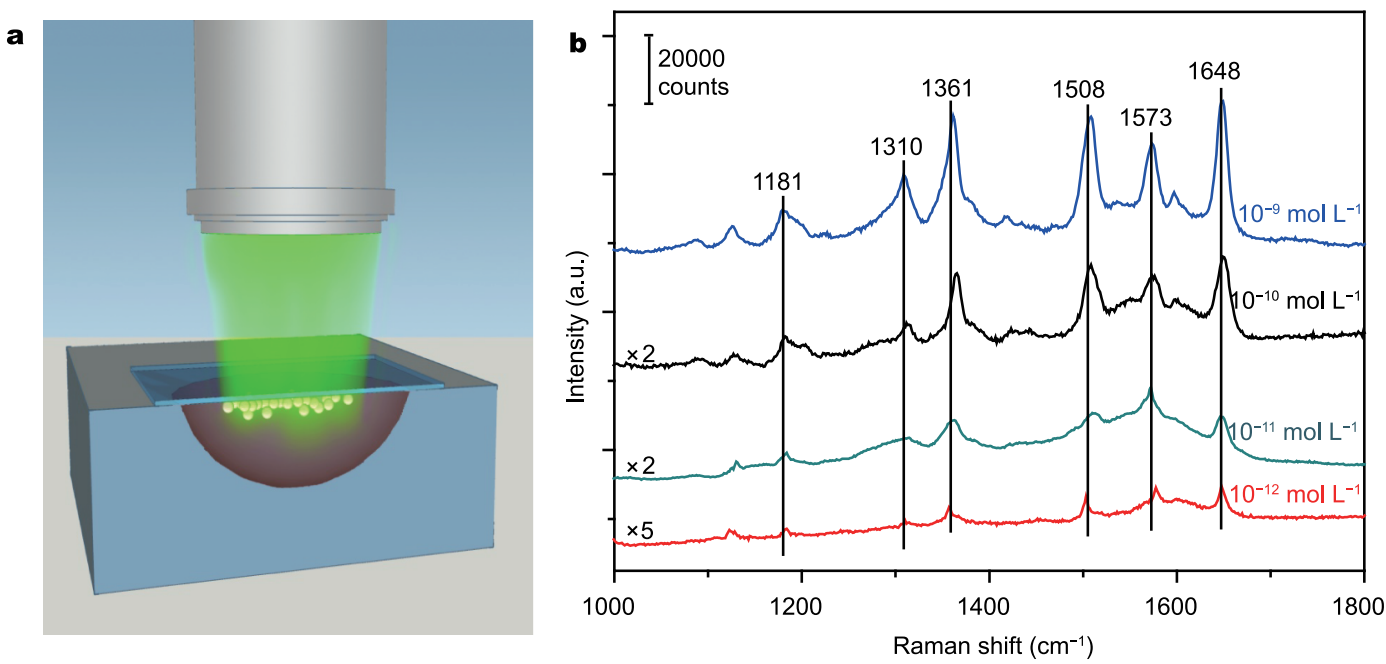

Figure 2 (a) Schematic of the measurement setup combining lens of Raman spectrometer, AgNLs/PDMS film, R6G solution and the sample holder; (b) SERS spectra of R6G with different concentrations $\left(1 \times 10^{-9} \sim 1 \times 10^{-12} \mathrm{~mol} \mathrm{~L}^{-1}\right)$ obtained by the same AgNL.

pherical dimple and its inner side contacting with R6G solution was washed with DI water and ethanol to remove the residue dye molecules. Before used for SERS detection of R6G with another concentration, washed AgNLs/PDMS film would be measured by Raman spectroscopy to insure that R6G molecules had been entirely removed (SI, Fig. S7 the blue curve). Then the cleaned SERS substrate was used to cover the R6G solution with another concentration to investigate the SERS sensitivity. With the ink mark on the top side of AgNLs/PDMS film, the same AgNL could be found even the R6G solution was changed. In this way, the realtime detection of aqueous solutions using AgNLs/PDMS elastomer as SERS substrate was evaluated (SI, S5).

Fig. 2b shows the collection of SERS spectra of R6G with different concentrations $\left(1 \times 10^{-9} \sim 1 \times 10^{-12} \mathrm{~mol} \mathrm{~L}^{-1}\right)$ obtained by the same AgNL. It can be noted that the SERS peak intensity decreases with the dilution of R6G solution. However, as the concentration reduced to $1 \times 10^{-12} \mathrm{~mol} \mathrm{~L}^{-1}$, the characteristic signal of R6G is still very clear, in which the pronounced bands at 1361,1508 , and $1648 \mathrm{~cm}^{-1}$ can be assigned to the aromatic $\mathrm{C}-\mathrm{C}$ stretching vibrations, meanwhile the weaker bands at 1310 and $1573 \mathrm{~cm}^{-1}$ are related to the $\mathrm{N}-\mathrm{H}$ in-plane bending modes. Meanwhile, we obtained the Raman spectra of AgNLs/PDMS film without contacting with R6G solution (Fig. S7), in which the peak around $\sim 710 \mathrm{~cm}^{-1}$ could be attributed to the PDMS substrate. As this Raman peak have no adverse impacts to the SERS signal of analytes, PDMS could be a feasible supporting material for SERS substrates. In order to quantitatively study the SERS enhancement ability of the AgNL/PDMS substrates, the enhancement factors (EF) were estimated ac- cording to the equation $\mathrm{EF}=\left(I_{\mathrm{SERS}} / I_{\mathrm{NR}}\right)\left(C_{\mathrm{NR}} / C_{\mathrm{SERS}}\right)[24,25]$. Normal Raman signals of R6G $\left(1 \times 10^{-2} \mathrm{~mol} \mathrm{~L}^{-1}\right)$ were obtained using the same hemispherical dimple without SERS substrate and the EFs were calculated at the main peaks of 1361 , and $1648 \mathrm{~cm}^{-1}$ (SI, S6). The average EF was $1.96 \times 10^{9}$, which is among the highest sensitivity of SERS substrates that achieves single-molecule detection [26-28].

Compared with the AgNPs colloids and the $\mathrm{Ag}$ nanowires assemble which barely detected R6G with the concentration of $1 \times 10^{-7} \mathrm{~mol} \mathrm{~L}^{-1}$ (SI, S7), the ultra-high SERS enhancement ability of this novel SERS substrate could be attributed to the novel structural three-dimensional high density "hot spots" of the as-prepared AgNLs and the special synthesis method without using surfactant. For the conventional AgNPs and AgNWs, the surfactants (sodium citrate, PVP) used in the synthesis might wrap on the surface of nanostructures and interfere with their contact to the analyte molecules. Meanwhile, the densely dispersed "hot spots" are hard to be achieved when the concentration of AgNPs or AgNWs solution is relatively low, thus leading to the low SERS sensitivity. In our study, the formation of Ag nano-beads of the nano-necklace is parallel with the consumption of SiNWs template. Small AgNPs are firstly deposited around the SiNWs, as the growth of AgNPs, the SiNWs are consumed and the Ag nano-beads are coming closer and stick together. In this way, the gaps of the nano-beads are very small and exist in all directions around the previous SiNWs. Thanks to the dense array of "hot spots" lying between the adjacent Ag nano-beads, the strong plasmonic coupling with dramatically amplified electromagnetic field could be obtained. 
Meanwhile, due to the absent of surfactant, the analyte can be directly contacted with the surface of AgNLs. As a result, our novel SERS substrate demonstrates ultra-sensitive detection ability, even though the laser and the SERS signal must penetrate through the PDMS film during measurement.

Although many different SERS substrates have been reported in previous literatures, one of the critical problems that still remain is the reproducibility and stability of the SERS substrates. In order to investigate the reproducibility of our SERS substrate, five different AgNLs/PDMS films were used to detect R6G solution with the concentration of $1 \times 10^{-9} \mathrm{~mol} \mathrm{~L}^{-1}$, and 6 different spots were investigated for each AgNLs. Fig. 3a displays five typical Raman spectra recorded from the measurements, which shows high consistency in shape and intensity. In addition, all the Raman peak intensities at $1650 \mathrm{~cm}^{-1}$ of these 30 points are analyzed in the histogram of Fig. 3b, in which all of the tested spots show SERS intensity of the same order of magnitude, which shows the excellent spot-to-spot and sample-to-sample reproducibility of AgNLs/PDMS substrates.
In the same testing setup, we also investigated the SERS signals of the AgNLs by Raman mapping (insert of Fig. 3b). The Raman mapping covers an area of $9 \mu \mathrm{m} \times 10 \mu \mathrm{m}$ with the intervals of each testing spots chosen as $0.5 \mu \mathrm{m}$. The intensity of the SERS signal of the band around $1650 \mathrm{~cm}^{-1}$ was plotted as a function of the coordinates. Compared with the microphotograph of AgNLs/PDMS film before Raman mapping (SI, Fig.S12), consistent SERS sensitivity is demonstrated along the AgNLs which confirms the high reproducibility of our SERS substrate. In order to investigate the stability of the AgNLs/PDMS film, after the study of reproducibility, the SERS active samples were stored in DI water for 7 months to investigate their stability with the same setup. Figs $3 \mathrm{c}$ and d show the typical Raman spectra and the peak intensity distribution of all the 35 spots chosen from 5 different AgNLs/PDMS substrates. Compared with the freshly-made samples, the intensities of Raman spectra show obvious reduction, however, the spectra are almost still in consistency in shape and the relative standard deviation of Raman peaks $\left(1650 \mathrm{~cm}^{-1}\right)$ of all the testing spots is $\sim 20 \%$, which shows good stability of the AgNLs and their
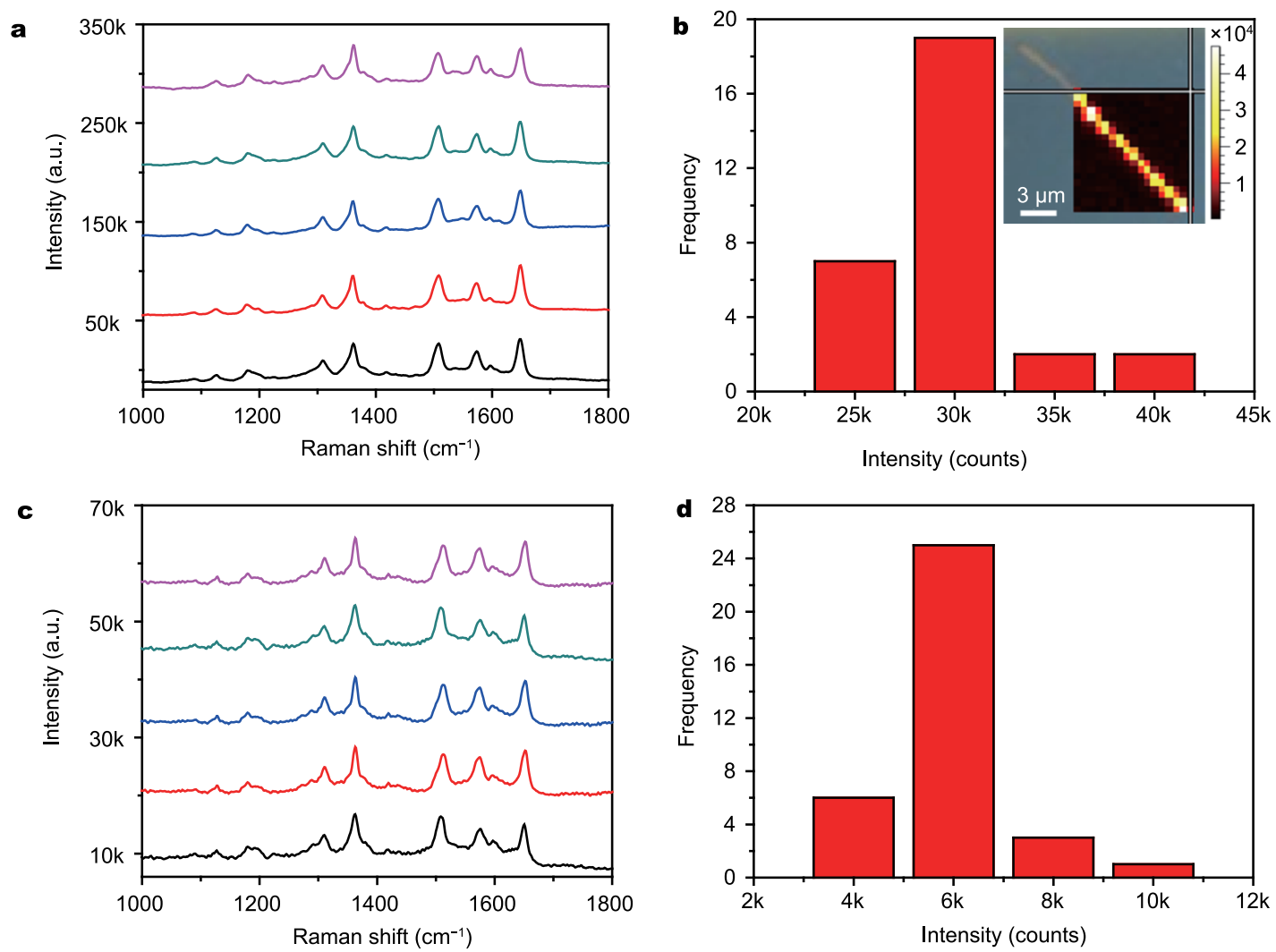

Figure 3 Typical Raman spectra recorded at five different AgNLs/PDMS films freshly made (a) and stored for 7 months in DI water (c). Histogram of intensity at $1650 \mathrm{~cm}^{-1}$ of all testing spots that obtained from freshly made AgNLs/PDMS substrates (b) and the water-stored samples (d), with R6G concentration of $1 \times 10^{-9} \mathrm{~mol} \mathrm{~L}^{-1}, 50 \times$ objective. The insert in (b) is the Raman mapping of AgNLs/PDMS that shows the intensity of SERS signal of the band around $1650 \mathrm{~cm}^{-1}$ as a function of the coordinates (R6G concentration $1 \times 10^{-9} \mathrm{~mol} \mathrm{~L}^{-1}, 100 \times$ objective). 
outstanding reproducibility even after 7-month storage in aqueous condition. These results mainly originate from the novel structure of the AgNLs which combines the advantages of AgNPs and AgNWs in a way that not only produces abundant micro-gaps between the nano-beads to amplify the electromagnetic field, but also strings and stabilizes the "hot spots" along the invisible core in 3-dimension which facilitates the reproduction of consistent SERS responses from different spots of different samples.

Using SERS techniques to identify the $\mathrm{pH}$ variation in aqueous condition is significant in biological and environmental fields. After modified by $\mathrm{pH}$-sensitive molecules pMBA, nanostructured-noble metals showed SERS sensitivity to $\mathrm{pH}$ changes of the surrounding solution [29]. In order to realize the reproducible, stable and real-time $\mathrm{pH}$ detection with high accuracy, we modified the AgNLs/PDMS film with pMBA (pMBA-AgNLs/PDMS) and investigated its $\mathrm{pH}$ response with the similar setup that detecting R6G (SI, S8). During the measurement, the pMBA-AgNLs/PDMS film contacted the testing solution with different $\mathrm{pH}$ by covering the solution-filled hemispheric dimple. 532-nm laser was shot through the SERS substrate from the $50 \times$ objective and the Raman spectra of pMBA under different $\mathrm{pH}$ values were obtained, in which the strong peak at $1590 \mathrm{~cm}^{-1}$ can be assigned to aromatic ring vibrations of $\mathrm{pMBA}$ and the $\mathrm{PH}$-sensitive weak Raman bands near 1395 and $1700 \mathrm{~cm}^{-1}$ indicate the presence of dissociated $\left(\mathrm{COO}^{-}\right)$and neutral $(\mathrm{C}=\mathrm{O})$ carboxylic groups (SI, Fig. S11). With the increasing of $\mathrm{pH}$, dissociation of the carboxyl group is promoted, leading to the intensity increase of $\mathrm{COO}^{-}\left(\sim 1395 \mathrm{~cm}^{-1}\right)$ and the suppression of $\mathrm{C}=\mathrm{O}\left(\sim 1700 \mathrm{~cm}^{-1}\right)$. However, in acidic environments, carboxylic groups turn to be nearly neutral and condense on the AgNLs' surface, thus intensifying the shoulder peak at $\sim 1700 \mathrm{~cm}^{-1}$ (SI, Fig. S11). It is reported that the signal intensity ratio of the stretching mode of the $\mathrm{COO}^{-}$groups and the constant aromatic ring vibrations mode $\left(1590 \mathrm{~cm}^{-1}\right)$ could be considered as labels to indicate $\mathrm{pH}$ changes [30]. Fig. 4a reveals the Raman intensity ratio as a function of $\mathrm{pH}$ value ranging from 4.0 to 9.0. For every $\mathrm{pH}$, ten spots from different AgNLs were detected and the AgNLs/PDMS substrates were rinsed with DI water before the measurement of different $\mathrm{pH}$. As can be seen, the SERS substrate based on the AgNLs can differentiate $\mathrm{pH}$ values over a wide range with a small standard deviation. Apart from the high sensitivity, the AgNLs/PDMS film also exhibits excellent repeatability and reversibility. Fig. $4 \mathrm{~b}$ displays the signal ratios of $\mathrm{COO}^{-}$ group and aromatic ring that are measured alternately in acid (pH 5.0) and alkaline ( $\mathrm{pH}$ 9.0) solutions, respectively. During the measurement, after testing in acid solution, the AgNLs/PDMS film was peeled from the hemispheric dimple and rinsed with DI water, then stuck to the other hemispheric dimple filled with alkaline solution to perform the corresponding measurement, and the cycle repeats for five times for each sample. As a result, the signal ratios obtained in different tests show negligible drift in the five cycles, which demonstrates the good stability and reproducibility of the AgNLs/PDMS substrates as $\mathrm{pH}$ detector. The highly reliable and repeatable $\mathrm{pH}$ response obtained by the AgNLs/PDMS elastomer could be attributed to the well-defined and novel assembly of the Ag nano-beads which originated from the same core string and huddled together to form a necklace. This unique structure could generate abundant SERS-active sites in three-dimension with sensitive and consistent SERS responses.
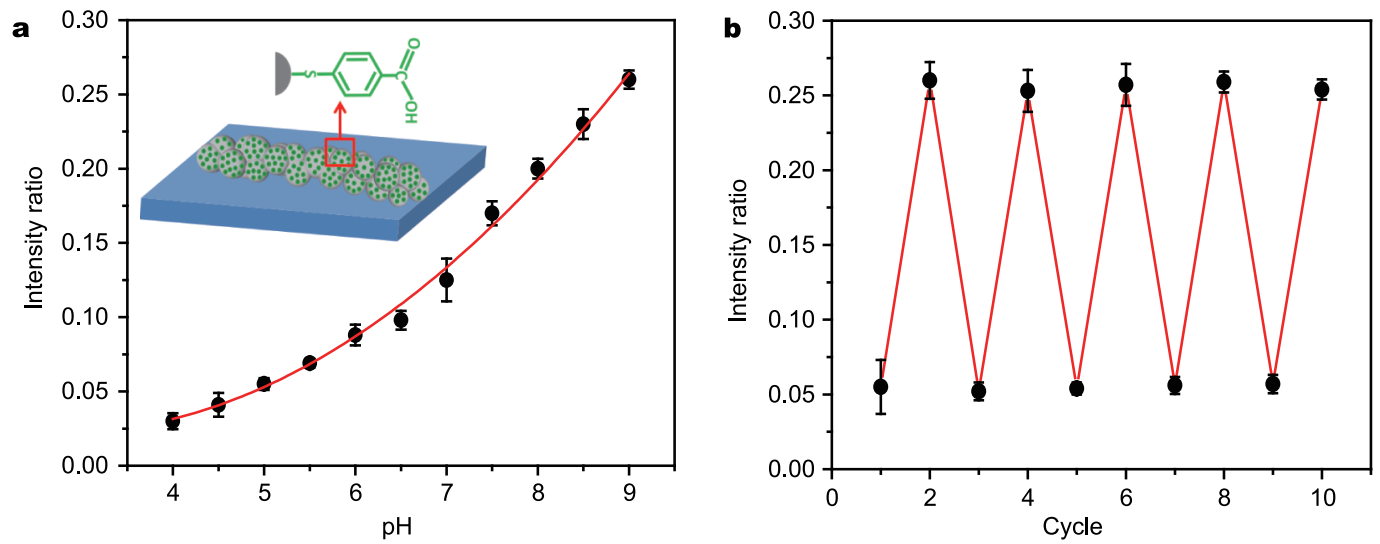

Figure 4 (a) Raman intensity ratio as a function of $\mathrm{pH}$ value ranging from 4.0 to 9.0. (b) Repeatable and reversible recording of the intensity ratios of $\mathrm{COO}^{-}$group and aromatic ring by alternate testing in acid $(\mathrm{pH} 5.0)$ and alkaline ( $\left.\mathrm{pH} 9.0\right)$ solutions. 


\section{CONCLUSIONS}

In summary, novel flexible SERS substrates based on AgNLs/PDMS were developed using SiNWs as sacrificing templates. With the stringing of dense "hot spot" in three-dimension, the AgNLs located on PDMS could work like the removable and reusable "tip" on the surface of analytes with different morphologies and conditions. Even across the PDMS supporting layer, the AgNLs also show ultra-high EF (as high as $10^{9}$ ), excellent reproducibility and long-term stability in an aqueous environment, which demonstrates the advantages of the AgNLs/PDMS substrate for high-resolution and compatible SERS detection. With further functionalizing with pMBA, the AgNLs/PDMS elastomer also reveals sensitive and consistent $\mathrm{pH}$ detection ability over the wide range of $\mathrm{pH}$ 4.0-9.0, indicating the wide applications in biological and environmental fields. The results suggest that the novel AgNLs-based flexible SERS substrate will provide new opportunities for sensitive, recyclable and versatile detection of chemical or biological substances in practical applications.

Received 25 April 2016; accepted 12 May 2016; published online 23 June 2016

1 De Angelis F, Gentile F, Mecarini F, et al. Breaking the diffusion limit with super-hydrophobic delivery of molecules to plasmonic nanofocusing SERS structures. Nat Photon, 2011, 5: 682-687

2 Chen HY, Lin MH, Wang CY, et al. Large-scale hot spot engineering for quantitative SERS at the single-molecule scale. J Am Chem Soc, 2015, 137: 13698-13705

3 Shi X, Li HW, Ying YL, et al. In situ monitoring of catalytic process variations in a single nanowire by dark-field-assisted surface-enhanced Raman spectroscopy. Chem Commun, 2016, 52: $1044-1047$

4 Stranahan SM, Willets KA. Super-resolution optical imaging of single-molecule SERS hot spots. Nano Lett, 2010, 10: 3777-3784

5 Zhao Y, Yang X, Li H, et al. Au nanoflower-Ag nanoparticle assembled SERS-active substrates for sensitive MC-LR detection. Chem Commun, 2015, 51: 16908-16911

6 Zhang H, Liu Y, Gao J, et al. A sensitive SERS detection of miRNA using a label-free multifunctional probe. Chem Commun, 2015, 51: $16836-16839$

7 Zhang L, Guan C, Wang Y, et al. Highly effective and uniform SERS substrates fabricated by etching multi-layered gold nanoparticle arrays. Nanoscale, 2016, 8: 5928-5937

8 Stamplecoskie KG, Scaiano JC. Light emitting diode irradiation can control the morphology and optical properties of silver nanoparticles. J Am Chem Soc, 2010, 132: 1825-1827

9 Cho WJ, Kim Y, Kim JK. Ultrahigh-density array of silver nanoclusters for SERS substrate with high sensitivity and excellent reproducibility. ACS Nano, 2012, 6: 249-255

10 Ren W, Fang Y, Wang E. A binary functional substrate for enrichment and ultrasensitive SERS spectroscopic detection of folic acid using graphene oxide/Ag nanoparticle hybrids. ACS Nano, 2011, 5: 6425-6433

11 Uzayisenga V, Lin XD, Li LM, et al. Synthesis, characterization, and
3D-FDTD simulation of $\mathrm{Ag} @ \mathrm{SiO}_{2}$ nanoparticles for shell-isolated nanoparticle-enhanced Raman spectroscopy. Langmuir, 2012, 28: 9140-9146

12 Kong X, Yu Q, Zhang X, et al. Synthesis and application of surface enhanced Raman scattering (SERS) tags of $\mathrm{Ag@SiO}{ }_{2}$ core/shell nanoparticles in protein detection. J Mater Chem, 2012, 22: 7767-7774

13 Yokota Y, Ueno K, Misawa H. Essential nanogap effects on surface-enhanced Raman scattering signals from closely spaced gold nanoparticles. Chem Commun, 2011, 47: 3505-3507

14 Duan H, Hu H, Kumar K, et al. Direct and reliable patterning of plasmonic nanostructures with sub-10-nm gaps. ACS Nano, 2011, 5: $7593-7600$

15 Liu M, Sun L, Cheng C, et al. Highly effective SERS substrates based on an atomic-layer-deposition-tailored nanorod array scaffold. Nanoscale, 2011, 3: 3627-3630

16 Bailo E, Deckert V. Tip-enhanced Raman spectroscopy of single RNA strands: towards a novel direct-sequencing method. Angew Chem Int Ed, 2008, 47: 1658-1661

17 Klingsporn JM, Jiang N, Pozzi EA, et al. Intramolecular insight into adsorbate-substrate interactions via low-temperature, ultrahigh-vacuum tip-enhanced Raman spectroscopy. J Am Chem Soc, 2014, 136: 3881-3887

18 Liu GL, Lee LP. Nanowell surface enhanced Raman scattering arrays fabricated by soft-lithography for label-free biomolecular detections in integrated microfluidics. Appl Phys Lett, 2005, 87: 07410

19 Weisse JM, Kim DR, Lee CH, et al. Vertical transfer of uniform silicon nanowire arrays via crack formation. Nano Lett, 2011, 11: 1300-1305

20 Peng $\mathrm{K}$, Lu A, Zhang R, et al. Motility of metal nanoparticles in silicon and induced anisotropic silicon etching. Adv Funct Mater, 2008, 18: 3026-3035

21 Wang $\mathrm{H}$, Zhang $\mathrm{X}$, Meng $\mathrm{X}$, et al. Bulk preparation of $\mathrm{Si}^{-S i O}{ }_{x}$ hierarchical structures: high-density radially oriented amorphous silica nanowires on a single-crystal silicon nanocore. Angew Chem Int Ed, 2005, 44: 6934-6937

22 Shiu SC, Hung SC, Chao JJ, et al. Massive transfer of vertically aligned Si nanowire array onto alien substrates and their characteristics. Appl Surface Sci, 2009, 255: 8566-8570

23 Pevzner A, Engel Y, Elnathan R, et al. Knocking down highly-ordered large-scale nanowire arrays. Nano Lett, 2010, 10: 1202-1208

24 Habouti S, Mátéfi-tempfli M, Solterbeck $\mathrm{CH}$, et al. On-substrate, self-standing Au-nanorod arrays showing morphology controlled properties. Nano Today, 2011, 6: 12-19

25 Gupta S, Agrawal M, Conrad M, et al. Poly(2-(dimethylamino)ethyl methacrylate) brushes with incorporated nanoparticles as a SERS active sensing layer. Adv Funct Mater, 2010, 20: $1756-1761$

26 Fu Q, Zhan Z, Dou J, et al. Highly reproducible and sensitive SERS Substrates with Ag inter-nanoparticle gaps of $5 \mathrm{~nm}$ fabricated by ultrathin aluminum mask technique. ACS Appl Mater Interfaces, 2015, 7: 13322-13328

27 Khlebtsov BN, Khanadeev VA, Panfilova EV, et al. Gold nanoisland films as reproducible SERS substrates for highly sensitive detection of fungicides. ACS Appl Mater Interfaces, 2015, 7: 6518-6529

28 Lee WWY, Silverson VAD, Jones LE, et al. Surface-enhanced Raman spectroscopy of novel psychoactive substances using polymerstabilized Ag nanoparticle aggregates. Chem Commun, 2016, 52: 493-496

29 Kneipp J, Kneipp H, Wittig B, et al. One- and two-photon excited optical $\mathrm{pH}$ probing for cells using surface-enhanced Raman and 
hyper-Raman nanosensors. Nano Lett, 2007, 7: 2819-2823

30 cells using encapsulated fluorescent SERS nanotags. Small, 2010, 6: 618-622

Acknowledgments This work was financially supported by the National Natural Science Foundation of China (51503210), and by the Hundred Talents Program of the Chinese Academy of Sciences (CAS), and by the State Key Laboratory of Multiphase Complex Systems Open Foundation (MPCS-2013-C-01).
Author contributions Duan $\mathrm{C}$ and Wang $\mathrm{Y}$ proposed the idea and designed the experiments. Duan C performed all the experiments. Ren B helped with the synthesis and characterization of materials. Duan C wrote the paper with help from Ren B, Liu H and Chen Y. Wang Y supervised the project and helped in paper writing. All authors contributed to the general discussion.

Conflict of interest The authors declare that they have no conflict of interest.

Supplementary information Details of synthesis of SiNWs and AgNL/PDMS film, SERS detection procedure of R6G and $\mathrm{PH}$, calculation of EF, and synthesis methods of AgNPs and AgNWs are available in the online version of this paper.

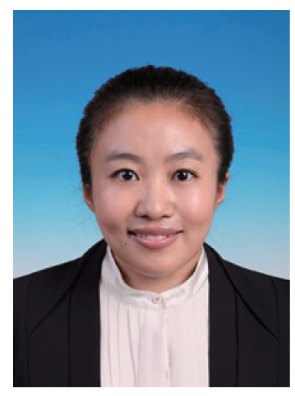

Chunyang Duan received her PhD degree from the Technical Institute of Physics and Chemistry, CAS in 2014. She is currently an assistant professor at Institute of Process Engineering, CAS. Her research interests mainly focus on nano-structured semiconductors for solar energy conversion and biochemical sensors.

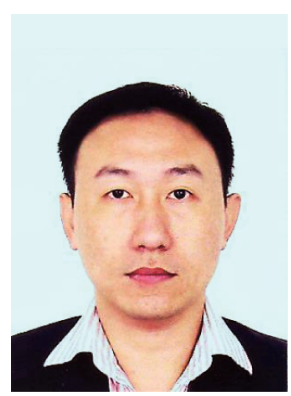

Wang Yu got his PhD degree from the Institute of Chemistry, CAS in 2008. He had been a research fellow in the Department of Chemistry, National University of Singapore since 2008. In 2013 he was selected by the "Hundred Talents" of CAS, as a full professor, in the Institute of Process Engineering, CAS. His research interests mainly involve manufacturing technology of $2 \mathrm{D}$ materials and their industrial applications, solar energy-transfer process and utilization.

\section{基于银纳米链的高重复性超灵敏尖端的柔性表面增强拉曼检测祄底}

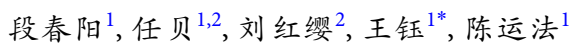

摘要 表面增强拉曼是一种可在液相中甚至细胞内实现对生物及化学分子的实时、原位、超灵敏检测的方法. 本文制备了高分子支撑的 银纳米链复合结构, 并将其作为超灵敏的表面增强拉曼检测衬底, 用于化学及生物检测. 通过将 “热点” 在三维方向上紧密集合, PDMS薄 膜上的银纳米链作为可移动、可重复利用的检测尖端, 在不同基底表面实现了具有超高的增强因子 $\left(10^{9}\right)$ 、优异的可重复性及长时间 $(7$ 个 月)的稳定性的化学检测. 通过进一步的修饰, 银纳米链/PDMS柔性衬底还可在 $\mathrm{pH}$ 值为 4.0 到 9.0 的范围内, 实现对溶液 $\mathrm{PH}$ 值的精确检测, 显 示出这种柔性衬底在生化及环境领域中的应用前景. 本研究为高效、稳定的表面增强拉曼柔性检测衬底的制备提供了新的途径. 